\title{
The characteristic taste of Eucommia leaf extract as the additive of a weight-loss and the constipation improvement is not associated with suppression of the feeding behavior of the fast rats with the extract
}

\author{
Hirotaka Oikawa ${ }^{1}$, Shouhei Miyazaki ${ }^{1}$, Wenping Zhang $^{2}$, Haruka Nishide ${ }^{1}$, Shouko Nakamichi ${ }^{1}$, Nami Morimoto-Kawaguchi ${ }^{1}$, Tetsuya

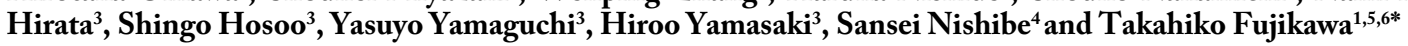 \\ ${ }^{1}$ Faculty of Pharmaceutical Sciences, Suzuka University of Medical Science, 3500-3 Minamitamagaki-cho, Suzuka-city, Mie, 513-8670, Japan \\ ${ }^{2}$ Faculty of Health science, Suzuka University of Medical Science, 1001-1, Kishioka, Suzuka-city, Mie, 510-0293, Japan \\ ${ }^{3}$ R\&D Center, Kobayashi Pharmaceutical Co., Ltd. 1-30-3 Toyokawa, Ibaraki-city, Osaka 567-0057, Japan \\ ${ }^{4}$ School of Pharmaceutical science, Health Sciences University of Hokkaido, 1757 Kanazawa, Tobetsu-cho, Ishikari-gun, Hokkaido, 061-0293, Japan \\ ${ }^{5}$ Division of Clinical Pharmacy and Pharmaceutical Sciences, Graduate School of Pharmaceutical Sciences. Suzuka University of Medical Science, 3500-3 \\ Minamitamagaki-cho, Suzuka-city, Mie, 513-8670, Japan \\ ${ }^{6}$ Mie University Graduate School / Faculty of Medicine, 2-174 Edobashi Tsu-city, Mie, 514-8507, Japan
}

\begin{abstract}
Du-Zhong tea, made from Eucommia ulmoides leaves, has been reported to provide an anti-hyperlipidemic effect by reducing visceral fat and suppressing elevated triglyceride and cholesterol levels in blood. In addition, ingestion of Eucommia leaf extract (ELE) has been reported to reduce food consumption by suppressing the physical activity of the rat at rest time. However, it was not known if ELE affects water consumption or how food consumption might be related to the taste of ELE. Therefore, this study investigates those effects and compares them with the production of rat feces. Nine-week-old Sprague-Dawley male rats were preliminarily reared for 1 week and then divided into two experimental groups that were fed a high-fat (35\% fat) diet (HFD): (1) rats supplemented with 5\% ELE (HFD-ELE-5\%) and (2) rats fed only a HFD (control group). We also analysed water consumption of rats in these two treatments for 1 week. We found that, during the rats' active phase (nighttime), water consumption increased under the HFD-ELE-5\% treatment, but that during the rats' inactive phase (daytime) phase, water consumption decreased under the HFD-ELE-5\% treatment. In another experiments, 9-week-old SD male rats were fasted for 48 hours and then divided into five groups fed a normal diet (ND): (1) rats with denatonium benzoate (DB) $0.001 \%$ to a ND (ND-DB-0.001\%), (2) rats with DB 0.1\% added to a ND (ND-DB-0.1\%), (3) rats with a ND amended with 5\% ELE (ND-ELE-5\%), (4) rats with a ND amended with 5\% Eucommia green leaf powder (ND-EGLP-5\%) and (5) rats with a ND as a control. Food consumption increased in every group relative to control. However, fecal production increased in the ND-ELE-5\% group and the ND-EGLP-5\% group. These results suggest that the taste of ELE does not likely affect food or water consumption. Thus, ELE indirectly suppresses food and water consumption by control of inactive time due to decreased motor activity during the inactive (sleeping phase). Therefore, we believe that ingesting ELE could be useful in preventing obesity and providing one component of an overall health management strategy.
\end{abstract}

\section{Introduction}

Eucommia ulmoides Oliver is a tall, deciduous, dioecious tree comprised of one family, one genus, and one species endemic to the Sichuan region of China. E. ulmoides is very rare in the wild and since ancient times, its bark has been consumed in China from over 2000 years ago for a variety of medicinal purposes, including to provide a nutritional supplement, to relieve low back pain and fatigue in legs, to improve liver and renal functions, and to relieve hypertension [1]. Its bark has also been used in Japan for medicinal purposes and it has been compounded as a Chinese herbal medicine to cure elbow joint disorders and hypertension.

Eucommia leaves are sometimes eaten and it has been reported that Eucommia decocted from tea leaves has an anti-hyperlipidemic effect that reduces visceral fat and reduces elevated triglycerides and cholesterols levels in the blood [2]. These effects may be attributable to the ingredients contained in its leaves. One report indicates that ingestion of Eucommia leaf extract (ELE) decreases plasma and liver
Correspondence to: Fujikawa T, Laboratory of Molecular Prophylaxis and Pharmacology, Faculty of Pharmaceutical Sciences, Suzuka University of Medical Science, Minamitamagaki-cho, Susuka, Mie 513-8670, Japan, E-mail: fujikawa@suzuka-u.ac.jp

Key words: Eucommia leaf extract (ELE), water consumption, taste of ELE, denatonium benzoate

Special Issue: Application to prophylactic pharmacology of food

Hirotaka Oikawa

Assistant professor

Department of Pharmaceutical Sciences

Suzuka University of Medical Science

Japan

Takahiko Fujikawa

Professor

Suzuka University of Medical Science Japan

Received: November 20, 2017; Accepted: December 01, 2017; Published: December 06, 2017 
Oikawa H (2017) The characteristic taste of Eucommia leaf extract as the additive of a weight-loss and the constipation improvement is not associated with suppression of the feeding behavior of the fast rats with the extract

lipids by suppressing fatty acid and cholesterol biosynthesis in the liver $[3,4]$. It has been shown that in rats fed a high-fat diet (HFD), ingestion of ELE or green leaf powder from Eucommia (EGLP) markedly prevents gaining weight and reduces the production of white fat tissue [5]. It has been suggested that this anti-obesity effect of ELE and EGLP is obtained by improving insulin resistance and hyperlipidemia by regulating the secretion and regulation of adipocytokines (which are dependent on visceral fat accumulation) [5]. In addition, ingestion of ELE prevented fat from accumulating in rates when they were fed high doses of fructose [6]. In addition, in rats that had undergone ovariectomy surgery, ingestion of ELE prevented the onset of osteoporosis and fat accumulation [7,8]. Furthermore, it has also been reported that ingestion of ELE enhances the gene expression of molecules related to fat oxidation [9]. We expect that the intake of ELE could improve and prevent many diseases linked to modern lifestyles, such as hypercholesterolemia, hypertension, and metabolic syndrome. Therefore, we believe that ELE could be used as a supplement to counteract such lifestyle diseases.

We have already reported on the changes in metabolic activity and fat oxidation that occurs from ingesting the ingredients contained in Eucommia leaves for rats [5] and that test rats reduced food consumption and generally became more inactive at inactive (daytime) phase [10]. These findings suggest that ELE ingestion can control of behavioural changes that inhibit caloric intake by increasing inactive time by stimulating increased physical activity in test rats. In preliminary experiment, we confirmed that ELE ingestion induced the protein expression of the neurotrophic factors in the rat central nervous system (CNS) (data not shown). The representative neurotrophic factor, brain-derived neurotropic factor (BDNF) is known to contribute to maturation of neurons and various brain functions as a regulation of food consumption. In addition, mutations in the BDNF gene are associated with obesity, and BDNF has potent inhibitory effects on eating and body weight [11]. Consequently, these finding suggests that consumption of Eucommia leaves stimulates the CNS. Therefore, to examine the potential influence of Eucommia extract on the CNS, this study quantifies daily water consumption as an indicator of the influence of Eucommia on the CNS as it relates to the stimulation of physical activity. In addition, because we had observed that ELE ingestion suppress appetite [10], we tested how the taste of ELE might be one factor affecting appetite suppression. To do this, we added a bitterant to rat feed and compared the subsequent behaviours of rats fed ELE supplemented feed and bitterant supplemented feed.

\section{Material and Methods}

\section{Animal metabolism cage}

We used an MK-5000RQ metabolic measurement system (Muromachi Kikai Co., Ltd.) to measure the metabolism of small animals. To examine the effect of ELE ingestion on rat's water consumptions had been recorded the scale of the water bottle for measurement in the metabolism cage each day from the active phase (19:00-07: 00), and the inactive phase (13:00-19:00 and 07:00-11:00). And the cage has wire mesh in the floor that allowed feces to drop through.

\section{Animals}

The rats used for this study were purchased from Japan SLC. Nineweek-old Sprague-Dawley (SD) male rats were reared for one week prior to the study and then used for the experiments, beginning at 10 weeks of age. The experiments were conducted in accordance with the animal-experiment guidelines established by the National Institutes of Health and the Suzuka Medical University Animal Experimental Ethics Committee, both of which approved the experimental plan.

\section{Feeding regimes}

According to our 2010 report [5], the anti-obesity effect of ELE and the anti-metabolic syndrome effect for rats fed a HFD were significantly observed compared with in rats fed a ND [5]. Therefore, in our ELE ingestion study, we used a HFD as the standard (basic) feeding regime for the experiment [5]. The basic HFD feed used in this study was purchased from Oriental Yeast Co., Ltd. as "MF powder" (containing $35 \%$ lard). This feed was supplied to rearing rats for one week after they had been acquired. After the one-week-long preliminary rearing period, a HFD diet containing 5\% ELE (HFD-ELE-5\%) was fed to one test group. In contrast, only a HFD was fed to a control (HFD-cont) group. (Kobayashi Pharmaceutical Co., Ltd provided the ELE used in this study.)

\section{Preparation of ELE}

We used Eucommia leaves collected in the Sichuan District of China. To prepare the ELE, fresh Eucommia leaves were steamed at $100-110^{\circ} \mathrm{C}$ and then dried and roasted. Quantities of $2 t$ of roasted Eucommia leaves were steeped in $10 \mathrm{t}$ of hot water at $90^{\circ} \mathrm{C}$ for $1 \mathrm{~h}$ and then the extract was then filtered and concentrated. The concentrate was allowed to stand for one day and then the concentrate was filtered and concentrated again, vacuum-dried, and powdered (yield: 18\%), as previously described [10].

\section{Conditions for rearing and water analysis}

The light-dark cycle of the rat rearing room was in the light phase from 7 am to $7 \mathrm{pm}$, and in the dark phase for remainder of each day. Because a rat is a nocturnal animal, the light phase is its inactive phase and the dark phase is its active phase. Care was taken to prevent the behaviour of rats in adjacent gauges from influencing one another. Furthermore, in order to prevent the scent of the wood chips covering the floors of the experimental areas from influencing behaviour, wood chips were not replenished or removed during the period of the experiments. The period of water consumption observation was one week after the rats had finished their period of preliminary rearing [10]

\section{Experiment on administrating bitter taste}

We had observed a decline in food consumption in rats fed food supplemented with 5\% ELE relative to control [10]. However, we did not examine whether or not the decline was caused by an unpalatable taste in ELE-treated food. Therefore, to test palatability in this study, as one of our feed treatments, we mixed a bitterant with ND in feed in order to provide a baseline against which the rat's taste for ELE could be examined, using relative food consumption as the index. Feed consumption was also examined comparing daily mass of food consumed and daily mass of feces produced. The denatonium benzoate (DB) was used to prevent ingestion of feed in the normal (basic) diet (ND)-DB group because rats can sense bitterness, even at concentrations as low as $10 \mathrm{ppb}$. This DB is generally used at a concentration of $0.001 \%$ to prevent accidental swallowing of fragrances. Nine-week-old SD male rats that had reared for one week were not fed for 48 hours. Then, we divided those rats into five experimental groups, each of which was fed for one hour on one of five diets: (1) ND (ND-Cont) only (the control group), (2) ND amended with 5\% ELE (ND-ELE 5\%), (3) ND amended with 5\% EGLP (ND-EGLP-5\%), (4) ND amended with $0.001 \%$ DB (ND-DB-0.001\%) and (5) ND amended with $0.1 \%$ 
DB (ND-DB 0.1\%). During this experiment, we examined changes in food intake, amount of feces produced, and ratio of feces/food intake. The food consumption was majored by electronic scale of MK-5000RQ metabolic measurement system. In addition, we compared the NDEGLP-5\% treatment with the ND-ELE-5\% treatment. The feces were collected from bottom of the MK-5000RQ cages, and then feces were weighted the amount by electronic scale.

\section{Statistical analysis}

Results of data analyses were expressed as the mean \pm S.E. (standard error) and statistical significance was determined with a one-way analysis of variance followed with a Dunnett T3-test, Dunnett t-test and/or a Games-Howell post-hoc test.

\section{Results}

\section{Effect of ELE on water consumption}

Effects of the HFD-ELE-5\% were examined relative to amount of water consumed, which was assumed to be an indicator of thirst. In the HFD-ELE-5\% group, daily water intake slightly increased insignificantly from Day 2 onward relative to the HFD-cont group (Figure 1). When daily water consumption was segregated into the active phase and the inactive phase, water consumed generally increased insignificantly during the dark phases (active period for rats) from Day 1 onward in the HFD-ELE-5\% ingestion group. However, the amount of water consumed significantly increased relative to the HFD-cont group after Day 6 onward (Figure 2). In contrast, during the light phases (inactive period for rats), HFD-ELE-5\% ingestion group showed a decline relative to the HFD-cont group in daily water intake from the Day 1 of the experiment onward. A significant decline in water intake was observed from Day 4 onward in the HFD-ELE-5\% ingestion group relative to the HFD-cont group (Figure 3).

\section{Effect of ELE taste on food intake}

We observed an increase in food intake per hour in the ND-ELE 5\% ingestion group relative to the ND-Cont group. In addition, food intake per hour significantly increased in the ND-EGLP-5\% ingestion group relative to the ND-Cont group. Furthermore, food intake per hour was significantly higher in the ND-DB-0.001\% ingestion group and in the ND-DB- $0.1 \%$ ingestion group relative to the ND-Cont group (Figure

\section{Daily water consumption} $(13: 00-11: 00)$

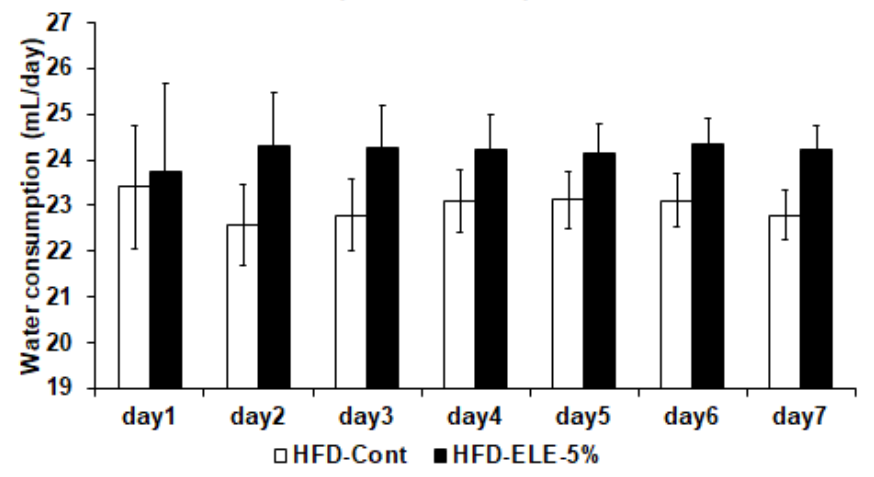

Figure 1. Effect of Eucommia leaf extract (ELE) ingestion on rat's daily water consumption: The rat's water consumption was measured by water bottle scale in metabolic cage. And the graph shows the daily water consumption in the HFD-control group and HFD-ELE-5\% group. Throughout the observation of 1-week period, the water consumption of the $5 \%$ ELE ingestion group modestly increased compared to the control group. Values are expressed in mean \pm SEM from 3 to 8 independent rats.

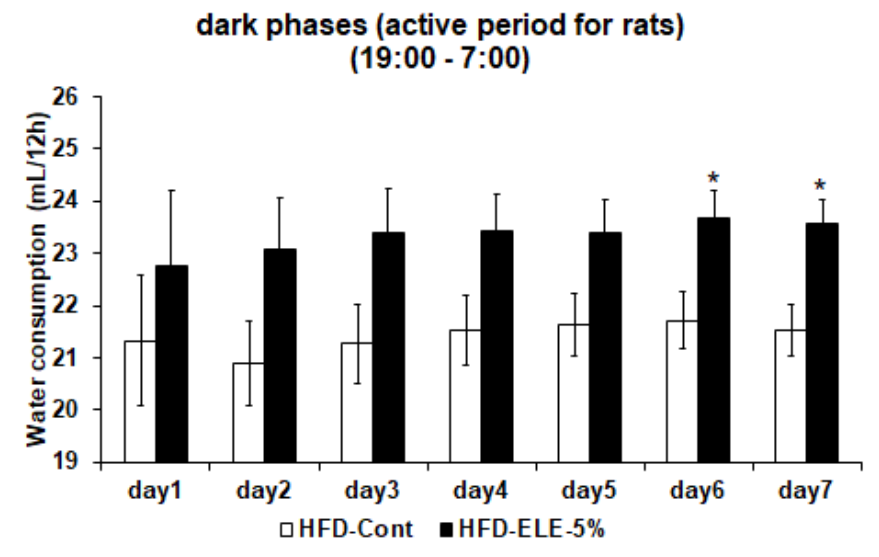

Figure 2. Effect of ELE ingestion on water consumption of rat's active phases: The water consumptions of the dark phases (active period for rat) from 19:00 to 7:00 were measured by water bottle scale in metabolic cage. And the graph shows the dark phases (active period for rat) water consumption in the HFD-control group and the HFDELE-5\% group. The water consumption of the 5\% ELE ingestion group modestly increased compared to the control group from day 1 to day 5. In addition, the water consumption significantly increased from day 6 to day 7 . Values are expressed in mean \pm SEM from 3 to 8 independent rats. ${ }^{*} \mathrm{P}<0.05$ vs. HFD-control experiment (Dunnett $\mathrm{T} 3$ test).

Light phases (inactive period for rats) $(13: 00-19: 00+7: 00-11: 00)$

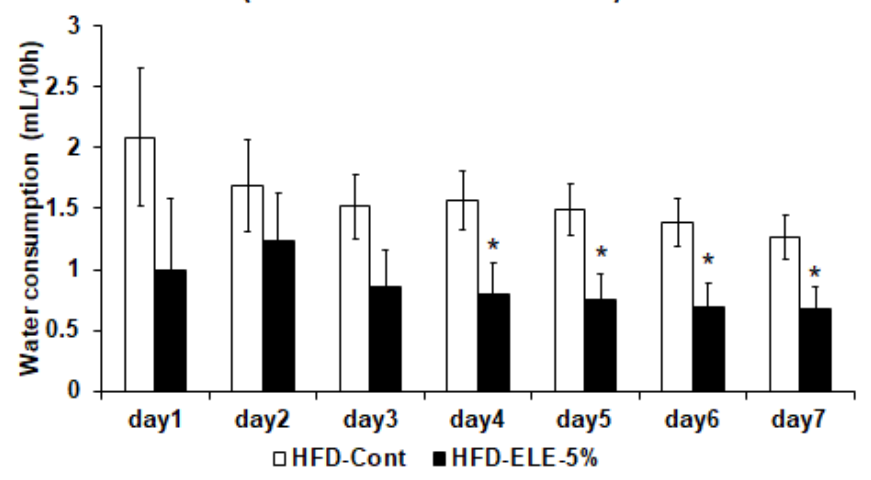

Figure 3. Effect of ELE ingestion on water consumption of rat's inactive phases: The water consumptions of the light phases (inactive period for rat) from 13:00 to 19:00 and from 7:00 to 11:00 were measured by water bottle scale in metabolic cage. And the graph shows the light phases (inactive period for rat) water consumption in the HFD-control group and the HFDELE-5\% group. The water consumption of the 5\% ELE ingestion group modestly decreased compared to the control group until day 3 . In addition, the water consumption significantly decreased from day 4 to day 7 . Values are expressed in mean \pm SEM from 3 to 8 independent rats. ${ }^{*} \mathrm{P}<0.05$ vs. HFD-control experiment (Dunnett $\mathrm{t}$ test, Dunnett $\mathrm{T} 3$ test).

4). Subsequently, when examining the amount of feces produced, we observed an increase in the amount of feces produced in the NDELE-5\% ingestion group. In the ND-EGLP-5\% ingestion group and in the ND-DB- $0.001 \%$ ingestion group, fecal production was significantly higher than in the ND-Cont group. The fecal production in the highlyconcentrated ND-DB ingestion group was the same as in the ND-Cont group (Figure 5). Also, when examining differences among treatments using the ratios of fecal production to food intake, we observed an increase in the the ratios of fecal production to food intake in the NDELE-5\% ingestion group. In the ND-EGLP-5\% ingestion group, the ratios of fecal production to food intake was significantly higher than in the ND-Cont group. In contrast, the ratios of fecal production to food intake was significantly less in the ND-DB-0.001\% ingestion group than in the ND-Cont group. Likewise, the ratios of fecal production to food intake was markedly less in the ND-DB-0.1\% ingestion group than in the ND-Cont group (Figure 6). 


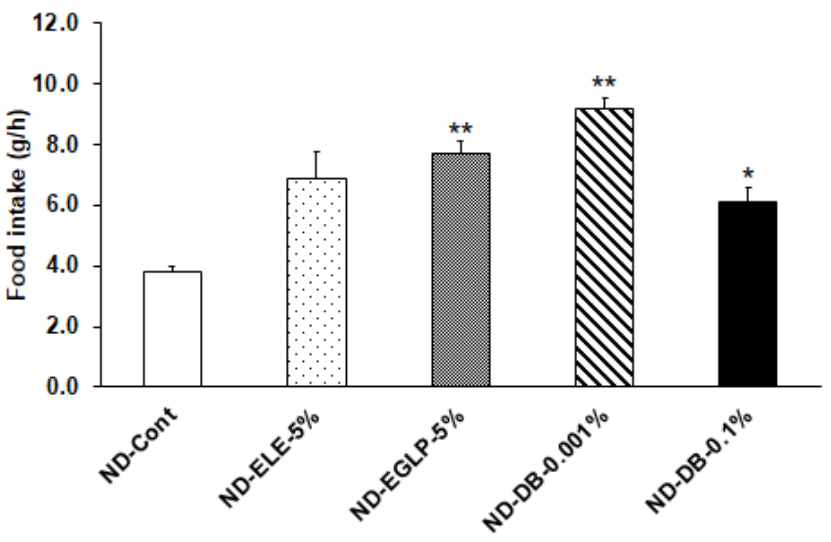

Figure 4. Effect of ELE taste and bitterant on food intake: Nine-week-old rats were fasted for 48 hours for a feeding test after one week of preliminary rearing. Then, rats were divided into five experimental groups as follows: ND-Cont, ND-ELE-5\%, ND-EGLP-5\%, NDDB- $0.001 \%$, and ND-DB- $0.1 \%$. These rats were fed for 1 hour. And the food consumption was measured by electronic scale of MK-5000RQ metabolic measurement system. The foo intakes significantly increased in the ND-ELE-5\%, ND-EGLP-5\% and ND-DB- $0.001 \%$ groups compared to the ND-Cont group. Values are expressed in mean \pm SEM 8 independent rats. ${ }^{*} \mathrm{P}<0.05$ vs. ND-Cont, ${ }^{*} * \mathrm{P}<0.01$ vs. ND-Cont experiment (Dunnett T3 test).

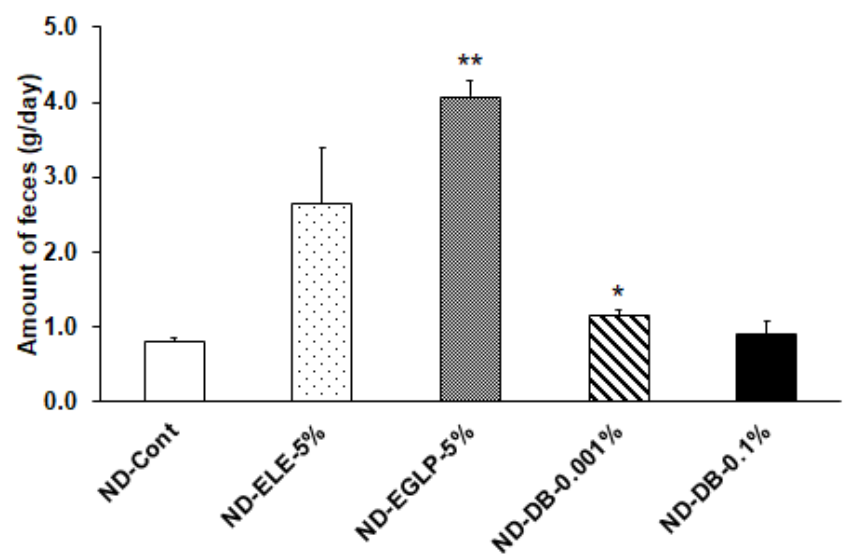

Figure 5. Effect of ELE taste and bitterant on feces amount: After 48 hours of fasting, the rats fed each subject diet for 1 hour were examined for the amount of feces per day. The amount of feces per day significantly increased in the ND-EGLP- $5 \%$ and the NDDB- $0.001 \%$ group compared to the ND-Cont group. Values are expressed in mean \pm SEM 8 independent rats. $* \mathrm{P}<0.05$ vs. ND-Cont, ${ }^{* *} \mathrm{P}<0.01$ vs. ND-Cont experiment (Dunnett T3 test).

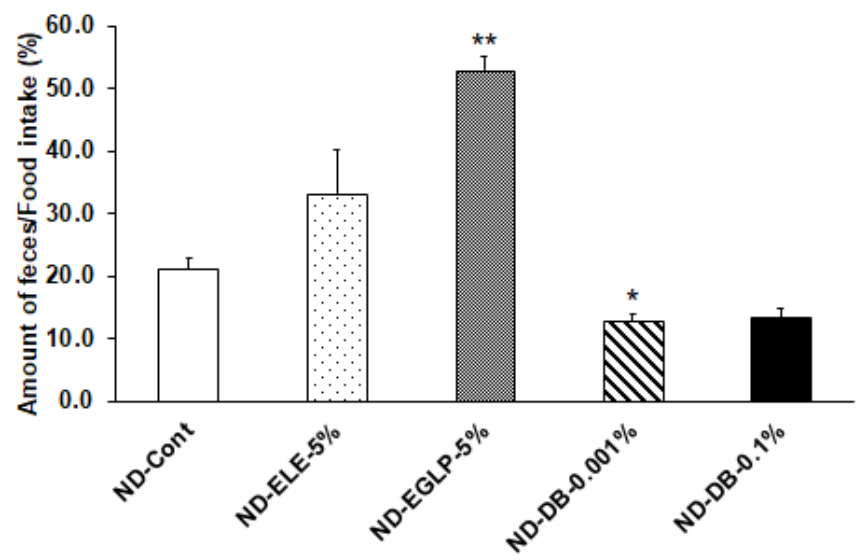

Figure 6. Effect of ELE taste and bitterant on ratio of feces amount/food intake: The ratio of feces amount/food intake was calculated by the consumption of each subject diet for 1 hour and the amount of feces per day. The ratio of feces amount/food intake significantly increased in the ND-EGLP-5\% compared to the ND-Cont group, while the ratio of feces amount/food intake significantly decreased in the ND-DB- $0.001 \%$ group. Values are expressed in mean \pm SEM 8 independent rats. ${ }^{*} \mathrm{P}<0.05$ vs. ND-Cont, ${ }^{*} * \mathrm{P}<0.01$ vs. NDCont experiment (Dunnett $\mathrm{T} 3$ test).

\section{Discussion}

We previously confirmed that rats consuming a HFD amended with 5\% ELE reduced their daily food consumption by inactivation of locomotor activity relative to a control group fed a diet without ELE [10]. As a result of our water consumption experiment, we found that it is consistent with the result of food intake behavior by HFD-ELE-5\% ingestion. Previous studies have reported that ingesting ELE suppresses fatty food intake and increases lipid metabolism, which together greatly reduces body weight by inhibiting the activity of the part of the parasympathetic nervous system that controls gastrointestinal tract functions $[12,13]$. However, the authors of these studies emphasised that the slight suppression of food intake that occurs in rats after giving them a high dose of ELE is the primary cause for weight reduction, while the metabolism-promoting effect of ELE is a secondary cause $[5,14]$. Thus, a study of metabolites in urine and feces might shed light on what is happening biochemically. Whereas, we preliminarily confirmed that ELE ingestion increased the protein expression of the neurotrophic factors in the rat hypothalamus (data not shown). The hypothalamus is a brain region that regulates homeostasis by mediating endocrine function, autonomic function and behavioral function such as a thirst, hunger, circadian rhythm and more [15]. In other words, ELE ingestion regulates appetite by acting on the CNS directly stimulation (such as the hypothalamus), and indirectly suppression (such as control of inactive time due to decreased motor activity). There has not been reported yet that ELE stimulation directly acts on the motor center, but in our report, HFD-ELE-5\% significantly increased spontaneous locomotor activity during active phase, and significantly decreased spontaneous locomotor activity during inactive phase [8]. We suspect that the anti-obesity effects are a direct result of our ELE treatments. The fact that water consumption was significantly lower during the rats' inactive (daytime) phase than control group in the HFD-ELE-5\% ingestion group suggests that there was a decline in such as search activity and grooming caused by the inactive time control. Namely, ELE ingestion induced inactive time extension in inactive (daytime) phase. In contrast, during the rats' active (nighttime) phase, there was a significant increase in water consumption (by thirsty) in rats of the HFD-ELE-5\% intake group. There was a possibility that the increase in water consumption in active (nighttime) phase was induced by the unpalatable of ELE which rats ate as bait, whereas the results of our study show that ELE induces fasting food intake behavior; this effect was also observed in rats provided EGLP. In addition, although fasting conditions, rats increased food intake of bitter mixed feed. Therefore, it is considered that the characteristic taste of ELE is not associated with suppression of the food intake behavior.

Meanwhile, under the HFD-ELE-5\% conditions, food intake behavior and water consumption behavior showed the same change, and water consumption did not affect food intake. Therefore, we believe that ELE components itself, but not the taste, affects both food and water consumption. Accordingly, we revealed that there appear to be factors other than taste that were responsible for increase in water consumption the HFD-ELE-5\% ingestion group during the rats' active period. This may be due to the difference in key components of ELE. Asperuloside, Geniposidic acid, chlorogenic acid are known as major components of ELE, and in addition to the anti-obesity effect, the CNS's effect such as preventing amyloid- $\beta$ induced cytotoxicity is known [16-18]. Therefore, future research should focus on the influence of various compounds absorbed from ELE by the intestinal tract. In addition, because ingestion of 5\% ELE in food increased the amount of feces produced relative to food intake, this suggests that ELE may improve defecation. Traditionally, it was believed that Du-Zhong 
Oikawa H (2017) The characteristic taste of Eucommia leaf extract as the additive of a weight-loss and the constipation improvement is not associated with suppression of the feeding behavior of the fast rats with the extract

tea has an action to relieve defecation, but it has not been scientifically studied. Therefore, the results of this study will be the first report in the world on the effect of defecation amelioration by ELE. This finding could be used ELE to prevent or alleviate constipation. However, further consideration such as whether ELE improves intestinal motility or raises the water content of feces seems to be necessary.

In summary, although ELE ingestion revealed an action to enhance fasting food intake activity, it is thought that food intake activity at satiety is suppressed. ELE ingestion enhances water consumption activity. And the characteristic taste of ELE is not considered to reduce the consumption of food. On the other hands, because ELE ingestion suppresses water consumption and appetite during a rat's inactive phase by inactive time extension effect, there is a possibility that ELE oral ingestion might also suppress midnight snacking and excessive drinking of juice in humans. In addition, ELE ingestion has improvement of defecation. Consequently, we believe that ELE could be useful for preventing obesity (by controlling appetite) and provide one component of an overall health management strategy.

\section{Acknowledgments}

This study was supported by a research grant from the Japanese Society of Eucommia. The Eucommia leaf extract samples used in this study were provided by KOBAYASHI Pharmaceutical Co., Ltd. (Osaka, Japan).

\section{Conflict of interest}

There are no conflicts of interest to declare.

\section{References}

1. Chinese Materia Medica Dictionary (1977) Jiangsu New Medical College. Shanghai: Shanghai Science and Technology Publishing House p. 1031

2. Hussain T, Tan B, Liu G, Oladele OA, Rahu N, et al. (2016) Health-Promoting Properties of Eucommia ulmoides: A Review. Evid Based Complement Altermat Med 2016: 5202908. [Crossref]

3. Choi MS, Jung UJ, Kim HJ, Do GM, Jeon SM, et al. (2008) Du-zhong (Eucommia ulmoides Oliver) leaf extract mediates hypolipidemic action in hamsters fed a high-fat diet. Am J Chin Med 36: 81-93. [Crossref]

4. Metori K, Ohashi S, Takahashi S, Tamura T (1994) Effects of Du-Zhong leaf extract on serum and hepatic lipids in rats fed a high-fat diet. Biol Pharm Bull 17: 917-920. [Crossref]
5. Fujikawa T, Hirata T, Wada A, Kawamura N, Yamaguchi Y, et al. (2010) Chronic administration of Eucommia leaf stimulates metabolic function of rats across several organs. Br J Nutr 104: 1868-1877. [Crossref]

6. Jin K, Amitani Y, Zamami Y, Takatori S, Hobara N, et al. (2010) Ameliorative effect of Eucommia ulmoides Oliv. leaves extract (ELE) on insulin resistance and abnormal perivascular innervation in fructosedrinking rats. J Ethnopharmacol 128: 672-678. [Crossref]

7. Zhang W, Fujikawa T, Mizuno K, Ishida T, Ooi K, et al. (2012) Eucommia leaf extract (ELE) prevents OVX-induced osteoporosis and obesity in rats. Am J Chin Med 40 : 735-752. [Crossref]

8. Zhang Z, Liu ZG, Li C, Hu SJ, Liu L, et al. (2009) Du-Zhong (Eucommia ulmoides Oliv.) cortex extract prevent OVX-induced osteoporosis in rats. Bone 45: 553-559. [Crossref]

9. Kobayashi Y, Hiroi T, Araki M, Hirokawa T, Miyazawa M, et al. (2012) Facilitative effects of Eucommia ulmoides on fatty acid oxidation in hypertriglyceridaemic rats. $J$ Sci Food Agric 92: 358-365. [Crossref]

10. Oikawa H, Nakamichi S, Nishide H, Kawaguchi M, Zhang W, et al. (2016) Engagement of the aroma in feeding suppression behavior of Eucommia leaf. Aroma Res 17: 74-79.

11. Gilland KE, Fox EA (2017) Effect of food deprivation or short-term Western diet feeding on BDNF protein expression in the hypothalamic arcuate paraventricula, and ventromedial nuclei. Am J Physiol Regul Integr Comp Physiol 312: R611-R625. [Crossref]

12. Horii Y, Tanida M, Shen J, Hirata T Kawamura N, et al. (2010) Effects of Eucommia leaf extracts on autonomic nerves, body temperature, lipolysis, food intake, and body weight. Neurosci Lett 479: 181-186. [Crossref]

13. He X, Wang J, Li M, Hao D, Yang Y, et al. (2014) Eucommia ulmoides Oliv.: ethnopharmacology, phytochemistry and pharmacology of an important traditional Chinese medicine. J Ethnopharmacol 151: 78-92. [Crossref]

14. Fujikawa T, Hirata T, Hosoo S, Nakajima K, Wada A, et al. (2012) Asperuloside stimulates metabolic function in rats across several organs under high-fat diet conditions, acting like the major ingredient of Eucommia leaves with anti-obesity activity. J Nutr Sci 1: e10. [Crossref]

15. Biran J, Tahor M, Wircer E, Levkowitz G (2015) Role of developmental factors in hypothalamic function. Front Neuroanat 9: 47. [Crossref]

16. Hirata T, Kobayashi T, Wada A, Ueda T, Fujikawa T, et al. (2011) Anti-obesity compounds in green leaves of Eucommia ulmoides. Bioorg Med Chem Lett 21: 17861791. [Crossref]

17. Tundis R, Loizzo MR, Menichini F, Statti GA, Menichini F (2008) Biological and pharmacylogical activeties of iridoids: recent developments. Mini Rev Med Chem 8: 399-420. [Crossref]

18. Zhou Y, Liang M, Li W, Li K, Li P, et al. (2009) Protective effects of Eucommia ulmoides Oliv. bark and leaf on amyloid $\beta$-induced cytotoxicity. Environ Toxicol Pharmacol 28: 342-349.

Copyright: (C2017 Oikawa H. This is an open-access article distributed under the terms of the Creative Commons Attribution License, which permits unrestricted use, distribution, and reproduction in any medium, provided the original author and source are credited. 